\title{
Inszenierung und Parodie männlicher Gewaltbereitschaft in La Haine und Allah Superstar
}

\author{
Laura Fuchs-Eisner
}

(C) Der/die Autor(en) 2016. Dieser Artikel ist eine Open-Access-Publikation.

Zusammenfassung Analysen der Repräsentation von Männlichkeit sind im Kontext der banlieue-Romane bzw. -Filme verhältnismäßig selten. Der Artikel widmet sich der Inszenierung von Männlichkeitsbildern in Mathieu Kassovitz' Film La Haine (Hass, 1995) und in Y. B.s Roman Allah Superstar (2003). Es wird der Frage nachgegangen, wie die Werke gängige bzw. klischierte Männlichkeitsbilder durch das übertreibende Element der Parodie hinterfragen, dadurch möglicherweise aber auch Darstellungen männlicher Gewaltbereitschaft zelebrieren.

Schlüsselwörter Allah Superstar · Hass (La Haine) · Y. B. Mathieu Kassovitz · Banlieue-Roman/-Film • Gewaltbereitschaft · Masculinity Studies · R. W. Connell • Parodie $\cdot$ Jugendliche im banlieue · Integration und Medien

\section{Representation and parody of male violence in La Haine and Allah Superstar}

\begin{abstract}
Studies on male identity are relatively rare in the context of banlieuenovels and -films. This article focuses on the representation of masculinity in Mathieu Kassovitz' film La Haine (Hate, 1995) and Y. B.'s novel Allah Superstar (2003). An analysis of parody as a subversive element in these,texts " shows that both of them question, but also reenact heavily stereotyped male roles through their parodistic play with symbols and scripts of masculinity.
\end{abstract}

Keywords Allah Superstar · Hate (La Haine) · Y. B. Mathieu Kassovitz • Banlieue-novel/-film · Male violence $\cdot$ Masculinity Studies · R. W. Connell · Parody · Banlieue youth · Integration and media

L. Fuchs-Eisner $(\bowtie)$

Kluckygasse 2/7-8, 1200 Wien, Österreich

E-Mail: laurafuchseisner@gmail.com 
Der Film La Haine (1995) von Mathieu Kassovitz ${ }^{1}$ und der Roman Allah Superstar (2003) von Y. B. ${ }^{2}$ können beide dem in Frankreich seit den 1990er-Jahren verstärkt rezipierten Genre des banlieue-Romans bzw. Films zugeschrieben werden. Wurde, besonders für den verwandten beur ${ }^{3}$-Roman, die,weibliche Identitätsproblematik mittlerweile ausführlich untersucht, so sind Analysen, die sich auf die Repräsentation von (jugendlicher) Männlichkeit konzentrieren, in diesem Kontext verhältnismäBig selten. Dieser Artikel widmet sich daher der Inszenierung von Männlichkeiten in La Haine und Allah Superstar und geht dabei auf die Rolle von Parodie als subversives Element ein. Der Fokus liegt auf der Repräsentation von Nachkommen von Migrant_innen und deren Erfahrungen als Teil der französischen (Migrations-) Gesellschaft der banlieue. Es soll herausgearbeitet werden, wie die Werke gängige bzw. klischierte Männlichkeitsbilder durch das übertreibende Element der Parodie hinterfragen - dadurch möglicherweise aber auch Darstellungen männlicher Gewaltbereitschaft zelebrieren.

Banlieue ist die allgemeine Bezeichnung für urbanisierte Gebiete außerhalb der Zentren französischer Großstädte. Ursprünglich neutrale Bezeichnung für Gebiete außerhalb der städtischen Bannmeile, ist sie heute Sammelbegriff für die Ballungsräume rund um die Zentren französischer Groß- bzw. Industriestädte. Seit den wiederholten Unruhen in französischen Vororten ab den 1980er-Jahren (vgl. Bachmann 2002, S. 329 ff.) und deren verstärkte Mediatisierung ist der Begriff überwiegend negativ konnotiert:

The „banlieues“ were to become a major category of journalistic and political discourse in the wake of rioting [...] during the early 1990s. Typically, these disturbances involved street battles between police and unemployed youth, often (though not always) of minority ethnic origin. [...] Events of this kind became synonymous with media coverage of the „banlieues“, which now came to appear not only as poor and ethnically marked but also as inherently lawless and violent spaces (vgl. Hargreaves 1999, S. 117).

In Vororten wie jenen, in denen die hier behandelten Texte spielen (Allah Superstar in Évry, La Haine in Chanteloup-les-Vignes), wurden ab den 1950er bis in die 1980er-Jahre Großwohnsiedlungen für Zuwanderer/-innen bzw. Immigrant/ -innen errichtet. Billige und schnell errichtete Sozialbauten ${ }^{4}$ sollten den eklatanten arbeitsmigrationsbedingten Wohnungsmangel der Industriestädte eindämmen und die unzumutbaren Baracken-Ghettos der mittellosen Arbeiterfamilien ersetzen. Mit

\footnotetext{
1 *1967 in Paris, als Sohn eines Filmemachers und einer Cutterin im Quartier Latin aufgewachsen. Sein jüdischer Vater war im Zuge der Ungarnaufstände nach Frankreich gekommen.

2 Yassir Benmiloud, *1968 in Algier als Sohn eines bekannten Psychotherapeuten. Er war während der 1990er Terrorjahre politischer Kolumnist und zeichnet seither seine Texte mit Y. B. Seit 1998 lebt er im Pariser Exil.

3 Beur/beurette - Begriff für die Nachkommen maghrebinischer Einwanderer in Frankreich. Auch verwendet für die Bezeichnung von Subkulturen (roman beur, cinéma beur); dabei aber, aufgrund seiner ethnisierenden Dimension, umstritten (vgl. Hargreaves 1999, S. 116).

4 Z. B.: Riesenbauten im Pariser Umland wie La Cité des 4000 in La Courneuve oder Les Indiens in Sartrouville (gebaut für 7000 Bewohner_innen).
} 
dem Abflauen des industriellen Wachstums ab den späten 1970er-Jahren, besonders aber während der Wirtschaftskrise in den 1990er-Jahren, wurden diese Zeichen der Moderne jedoch zu sozialen Brennpunkten (vgl. Bachmann 2002; Beaud 2003) gezeichnet von hoher (Jugend-)Arbeitslosigkeit ${ }^{5}$, (häuslicher) Gewalt, Kleinkriminalität und Spannungen unter den Bewohner_innen (vgl. Beaud 2003, 11 ff.). Wie im vorangehenden Zitat von Hargreaves angesprochen, waren sie seit den späten 1980er-Jahren wiederholt und teils überdurchschnittlich stark mediatisiert Schauplatz von Unruhen. Die jeunes des banlieues machten durch Vandalismus und Ausschreitungen gegen die Polizei - die spätestens 2005, als sie zur Ausrufung des état d'urgence führten, einen traurigen Höhepunkt erreichten - auf sich aufmerksam (vgl. Balibar 2007; Kokoreff et al. 2007).

Waren die banlieues zunächst sozial durchmischt, so änderte sich die Zusammensetzung jener Gebiete, die als problematisch galten, bis in die 1990er-Jahre drastisch. ${ }^{6}$ Die große Anzahl von Nachkommen der maghrebinischen Einwanderer in den quartiers sensibles ist dabei in der allgemeinen Wahrnehmung besonders präsent (Vincendeau 2005, S. 31). Wenn von Integrationsproblemen die Rede ist bzw. diese verstärkt mediatisiert werden, betrifft dies häufig die in den banlieues lebenden ,,beurs “ (O’Dowd-Smyth 2004, 316 f.). Diese sind somit Opfer einer doppelten Stigmatisierung aufgrund von Ethnie und Wohnort. Auf dieses Zusammenspiel von délit de faciès und délit d'adresse (vgl. Ottersbach 2004, S. 63) führt Étienne Balibar u. a. auch das hohe Frustrations- und Gewaltpotential der (männlichen) Jugend im banlieue zurück: „Each stigma prevents them from freeing themselves from the other, crushing their individual and collective futures“ (2007, S. 57 f.).

Ab 1985 gab es nicht zuletzt aus diesem Grund zunächst mit den beur-Romanen bzw. -Filmen, ${ }^{7}$ ab den 1990er-Jahren dann mit den banlieue-Filmen bzw. -Romanen verstärkt literarische und filmische Stimmen, die durch alternative Repräsentationen den mediatisierten Bildern differenziertere Darstellungen dieses sozialen Kosmos entgegenhalten sollten. So arbeitet sich laut Christine O'Dowd-Smyth die beurLiteratur ab den 1980er-Jahren an der kulturellen Hegemonie Frankreichs ab und bricht ,the silence of cultural and social exile“ (2004, S. 321).

La Haine und Allah Superstar werden hier, ob der von ihnen dargestellten sozialen Räume, als typische Vertreter des banlieue-Films bzw. -Romans gesehen. Die beiden Werke gehen auf narrativer Ebene auf aktuelle Probleme in der französischen Gesellschaft ein. La Haine begleitet die arbeitslosen jungen Männer Said, Vinz und Hubert, ein multiethnisches, multireligiöses Trio aus La Noë in Chanteloup-les-Vignes, durch einen Tag in ihrer cité und eine Nacht im Stadtzentrum von Paris. Der

\footnotetext{
5 Z. B. in Évry: Laut dem französischen Statistikamt INSEE lag die verzeichnete Arbeitslosenrate unter den 15-24-Jährigen 2012 bei 36,6\% (bei einem Mittelwert der französischen Städte von 25,2\%) (vgl. INSEE 2016).

${ }^{6}$ Für eine Zusammenfassung der sukzessiven „Ghettoisierung“ von Immigrant_innen und deren Nachkommen in manchen Vororten zwischen den 1970er und 1990er-Jahren vgl. Weil (1991, S. 390 ff.).

7 Die Bezeichnung beur-Roman ist, so wie die des banlieue-Genres, insgesamt allerdings zu problematisieren: Wählt ein Teil der betroffenen Künstler/-innen die Bezeichnung ganz bewusst, wehren sich andere gegen diese Klassifizierung, weil sie ihre Kunst weder ethnisiert (beur) noch ghettoisiert (banlieue) sehen wollen (vgl. Tarr 2005, S. 13, und Hargreaves 1999, S. 115). Hier wird der Begriff verwendet, um die Werke anhand des dargestellten Milieus zu charakterisieren.
} 
Film behandelt aus ihrer Perspektive Unruhen in französischen Vorstädten. Die Protagonisten sind dabei fiktiv, der Film verweist allerdings auf wahre Hintergründe. ${ }^{8}$ In Allah Superstar erzählt der 19-jährige beur Kamel Léon Hassani, Sohn eines Algeriers und einer Französin, seine Entwicklung vom Mitarbeiter einer FastfoodKette zum Stand-up-Comedian, der mit einer Show als Fake-Djihadist bekannt wird. Zum medialen Durchbruch verhilft ihm eine Fatwa; am Höhepunkt seiner Karriere sprengt er sich vor Publikum tatsächlich in die Luft. Der Roman thematisiert dabei weniger religiösen Fanatismus als viel eher die Perspektiven der beurs in Frankreich bzw. den Umgang der französischen Medien mit der religiösen und ethnischen Heterogenität Frankreichs.

Andererseits zeichnen sich beide Werke durch ihre auffällige Ästhetisierung des dargestellten sozialen Umfelds aus. La Haine bricht als Schwarz-Weiß-Film mit den Konventionen der 1990er-Jahre und ist bekannt für seine gekonnten Kameraeinstellungen und sein dichtes Netz an intermedialen Verweisen; Allah Superstar ist durchgehend im français contemporain des cités ${ }^{9}$ geschrieben und hat so stark satirisch-komischen Charakter, dass der Roman auch als „one man Show narratif“ bezeichnet wurde (vgl. Vitali 2011, S. 15). Beide verbinden einen markanten filmischen bzw. literarischen Stil mit Elementen von Satire und Parodie ${ }^{10}$, indem sie mit bekannten Bildern und Codes spielen und durch Überzeichnung ambivalente Botschaften vermitteln. Durch ihren bewussten Umgang mit den Möglichkeiten der Kunstformen Film und Literatur tun sich die beiden Werke innerhalb eines Genres hervor, dem häufig der Anschein des Dokumentarischen anhaftet. Die damit verbundenen Missverständnisse bei ihrer Rezeption werden später noch thematisiert.

In der Folge soll nun die Darstellung von marginalisierter Männlichkeit und Gewalt in La Haine und Allah Superstar untersucht werden. Die Überlegungen orientieren sich dabei am Konzept von R. W. Connell, die in Masculinities (1995) ein Modell von vier Typen von Männlichkeitsverhältnissen aufstellt. ${ }^{11}$ Interessant für die folgende Analyse sind dabei die hegemoniale und die marginalisierte Männlichkeit. Erstere steht für den im jeweiligen Kontext dominierenden Idealtypus, der symbolisch gesellschaftliche Macht innehat. Verkürzt ist dies für den,westlichen' Kontext: weiß, heterosexuell, beruflich erfolgreich, mächtig (vgl. Spindler 1999, S. 122). Dies

\footnotetext{
8 Der Film setzt mit Originalvideomaterial von Fernsehberichten über Unruhen in den Pariser Vorstädten ein. Auch der Fall von Abdel Ichaha, der im Film unter Polizeigewalt seinen tödlichen Verletzungen erliegt, hat wahre Hintergründe: Kassovitz begann das Drehbuch 1992 zu schreiben, als der Tod Makome M'Bowoles, eines jugendlichen Immigranten aus dem Kongo, der in Untersuchungshaft von Polizisten erschossen wurde, gewaltvolle Unruhen in den banlieues auslöste. Makomes Schicksal steht in einer Reihe von Todesfällen junger Männer, die auf polizeilichem Fehlverhalten beruhen (2005 zählte Ginette Vincendeau 300 seit 1981 (2005, S. 12)).

9 Der Linguist Jean-Pierre Goudaillier definiert diese vom Verlan (Verdrehung von Syntax und Vokalfolge) geprägte Umgangssprache als interlangue aus Standardfranzösisch und Lehnwörtern bzw. -strukturen aus dem maghrebinischen Arabisch, Berber- und Kreolsprachen, weiteren afrikanischen Sprachen etc. (2001, S. 6 f.).

10 Am Rande sei angemerkt, dass sich dieser Artikel auf die narratologische Definition von Parodie von Daniel Sangsue stützt; also jener der Parodie als einer spielerischen, komischen oder satirischen Transformation eines Ursprungstexts (Sangsue 1994, S. 5). Der Begriff,Text ‘ wird hier breit gedacht und umfasst neben filmischen und literarischen Vorlagen u. a. auch mediale Darstellungen und kollektive Vorstellungen.

11 Hegemony, Subordination, Marginalization und Complicity.
} 
impliziert die Existenz hierarchisch untergeordneter Typen wie der marginalisierten Männlichkeit, bei der laut Connell Aspekte von Klasse und *race besonders ins Spiel kämen. Marginalisierte Männer seien jene, die aus Gründen ihrer ethnischen oder sozio-ökonomischen Herkunft kaum eine Chance hätten, das hegemoniale Ideal der Mehrheitsgesellschaft zu erfüllen. Signifikante Arbeitslosenzahlen, städtische Armut und institutionalisierter Rassismus trügen essentiell zur Marginalisierung bestimmter Männergruppen bei (vgl. Connell [1995] 2005, S. 80). Dafür bildeten sich innerhalb der marginalisierten Gruppen alternative Code-Systeme aus: Ehre, Authentizität, Loyalität, Härte und Widerstand gegen die Staatsgewalt (Kriminalität, Zeit im Gefängnis ...) könnten somit Messlatten für interne Hierarchisierungen werden. Connell hat in ihrer Studie für die Gruppe der marginalisierten Männer die black community in den USA untersucht. Dass ihr Konzept auch auf andere Kontexte übertragen werden kann, zeigt u. a. eine Interviewstudie mit delinquenten jugendlichen Migranten in Deutschland von Susanne Spindler. Diese hält fest, wie die betroffenen Jugendlichen ,sich in einem permanenten Ringen um gesellschaftlich anerkannte und,normale` Männlichkeit befänden und versuchten, sich hegemonialer Männlichkeit anzunähern“ (2009, S. 121) bzw. ihre Ausgrenzung mit alternativen Codes der Hierarchisierung innerhalb ihres nächsten Umfelds zu kompensieren, die im Widerspruch zu den Normen der ausgrenzenden Instanz stehen und mit (der Zelebrierung von) Delinquenz verbunden sein können (vgl. Subkulturen wie Hip Hop, Gangster-Rap und Street Art).

Ein ähnliches Muster zeigt sich in den ausgewählten Werken. Beide thematisieren die Perspektivlosigkeit der männlichen banlieue-Jugend und die Konflikte innerhalb einer französischen Gesellschaft, die an einer jahrzehntelang mangelhaften Einwanderungs-, Integrations- und Wohnpolitik krankt. Beide Werke bedienen genau jenes Stereotyp machohaft agierender migrantischer Jugendlicher, das im Laufe der dieser Publikation vorausgehenden Konferenz mehrmals angesprochen wurde. Hier soll allerdings gezeigt werden, dass sie mit diesem Stereotyp spielen, es überzeichnen und damit auch in Frage stellen.

La Haine ist sicherlich der (international) bekannteste und finanziell erfolgreichste banlieue-Film. Er wird als Wende- und Höhepunkt des Genres - „,both harbinger and epitome of the film de banlieue" (Vincendeau 2005, S. 98) - gesehen (Tarr 2005, S. 2, 16). In der Szene ist der Film allerdings auch umstritten, wird doch immer wieder diskutiert, ob der Film nun tatsächlich alternative Visionen schaffe oder viel eher Klischees aufgreife, publikumswirksam ästhetisiere und sie durch seine Bekanntheit festschreibe (vgl. Vincendeau 2005, S. 69; Ruhe 2006, S. 134 f.). In La Haine ist das multiethnische, multireligiöse Trio Vinz, Hubert und Said eine recht bitter-ironische Anspielung auf den französischen Integrationsslogan blackblanc-beur ${ }^{12}$, präsentiert der Film doch alles andere als eine Erfolgsgeschichte des gesellschaftlichen Zusammenlebens: Die Protagonisten sind im Gegenteil (in un-

\footnotetext{
12 Dieser wird seit 1998, als eine multiethnische französische Nationalmannschaft die Fußball-Weltmeisterschaft gewann, zumeist medienwirksam mit positiver Konnotation für die ethnische Vielfalt der französischen Gesellschaft verwendet. In Frankreich wird die Fußballnationalmannschaft der Männer, in Anlehnung an die dreifärbige Flagge, auch équipe tricolore genannt. Die Verschiebung von bleu-blanc-rouge zu black-blanc-beur symbolisiert die verschiedenen Ethnien jener Spieler, die Frankreich 1998 den Weltmeistertitel einbrachten (vgl. Doering et al. 2002, S. 28).
} 
terschiedlichem Ausmaß) Schulversager, Drogendealer, Schläger, Vandalen, arbeitsund perspektivlos. Gleichzeitig sind sie allerdings auch loyale Freunde; Söhne, die auf Abwegen Geld nach Hause bringen; junge Männer, die für Ehre und die Frage der Authentizität den täglichen Straßenkampf bestreiten. Auffallend ist im Film die Inszenierung der hohen verbalen und physischen Gewaltbereitschaft und der sinnund ziellosen Aggressionen der jungen Männer auf der Straße, wo sie ständig in Konflikte mit der Polizei oder den Bewohnern des Pariser Stadtzentrums verwickelt sind. In diesem Punkt ist der Film äußerst ambivalent: Er inszeniert die gegenseitigen Provokationen zwischen desillusionierter, gewalttätiger Vorstadtjugend und ihrer Gegenseite (Polizei, Pariser Establishment, Journalisten). Die jungen Männer sind dabei so sinnlos gewalttätig und aggressiv, wie sie im Gegenzug mit Vorurteilen, Rassismen und Ausgrenzung konfrontiert werden. So beginnen sie mit Steinen um sich zu werfen, als Journalisten sie in der cité von geschlossenen Autos aus filmen und interviewen wollen (vgl. Kemner 2001, S. 38), oder randalieren, als sie Abdel $^{13}$ im Krankenhaus besuchen wollen und keinen Zutritt bekommen. Sie kontern Erniedrigung, Exklusionen und Machtlosigkeit mit Aggressionen und spiegeln damit Farhad Khosrokhavars (2003, S. 94) These, laut der mehrfache Entwertung von Jugendlichen verinnerlicht und in Form von Gewaltausbrüchen gesellschaftlich sichtbar würde (vgl. auch Ruhe 2006, S. 116 ff.).

In Allah Superstar ist Protagonist Kamel Hassani als Sohn eines algerischen Arbeiters und Bewohner des banlieues Évry laut Eigendefinition in doppeltem Sinne „d'origine difficile“ (Y. B. 2003, S. 9). Obwohl er rein theoretisch perfekt,akkulturiert' ist - er ist in Frankreich aufgewachsen und beweist im Zuge seiner Ich-Erzählung einen breiten, (auch) frankreichbasierten, (pop)kulturellen Verweishorizont ${ }^{14}$, , fühlt er sich im Abseits der französischen Gesellschaft. Als ,Franko-Araber' stünden ihm nur zwei Möglichkeiten offen: entweder Respekt, Ansehen und Geld durch Berühmtheit (wie sein Idol, der Franko-Marokkaner Djamel Debbouze) oder eine Nicht-Existenz als,banlieue-Nichts“: „Si tu prends un jeune d'origine difficile issu d'un quartier sensible [...] donc un Arabe ou un Noir, eh bien lui il a pas le choix: soit il est une star soit il est rien"15 (Y. B. 2003, S. 11). Dies ist eine Anspielung auf die Tatsache, dass Frankreich seine franko-maghrebinischen Schauspieler/-innen, Autor/-innen, Fußballspieler und Comedians sehr wohl mit Stolz präsentiert, Vorurteile gegenüber maghrebinischen Einwanderern dadurch allerdings nicht abnehmen. ${ }^{16}$ Der Erzähler Kamel konfrontiert die Le-

\footnotetext{
13 Abdel Ichaha stirbt im Film nach Auseinandersetzungen mit der Polizei (vgl. Fußnote 8). Für Vinz besteht nach seinem Tod Handlungsbedarf, hat er doch davor angekündigt, einen Polizisten zu erschießen, sollte sein Kumpel sterben.

14 So jongliert Kamel mit Verweisen auf die französische Fernsehwelt, Stand-up-Comedy, Rap und französische Politik, zitiert aber auch Frantz Fanon, Shakespeare und den Koran.

15 Meine Übersetzung: „Denk an einen mit schwierigem Hintergrund aus einem Problemviertel [...] also einen Araber oder einen Schwarzen, der hat keine Wahl: entweder ist der ein Star oder der ist ein Nichts." Diese Passage ist zudem ein gutes Beispiel dafür, wie Y. B. mit amtssprachlicher political correctness spielt.

16 Auch in diesem Punkt zeigt sich eine Parallele zu R. W. Connells Analyse ethnisch bedingter marginalisierter Männlichkeiten in den USA: „The fame and wealth of individual stars has no trickle-down effect; it does not yield social authority to black men generally“ (2005, S. 81).
} 
ser_innen konsequenterweise von Beginn an mit,ihren' Vorbehalten; etwa wenn er seine Religionszugehörigkeit folgendermaßen vorstellt:

Mais je sais, tu te demandes si je suis musulman pratiquement [sic!] comme mon père, si je fais la prière, le ramadan, les ceintures d'explosifs, les tournantes dans les mosquées-caves sur des mineures excisées par des imams sans papiers qui les attachent au minaret avec un foulard islamique malgré Sarkozy.

(Y. B. 2003, S. 16) ${ }^{17}$

In diesem Zitat zeigt sich einerseits Benmilouds Art und Weise, mit sprachlichen und kulturellen Vorstellungen zu jonglieren - wenn er etwa aus dem Adjektiv pratiquant das Adverb pratiquement macht und darin spielerisch den Unterschied zwischen einem ,praktizierenden Moslem“ und Moslem „fast so wie mein Vater“ verpackt oder er das Amalgam von Moslem und Terrorist bloßstellt, in dem er „la prière, le ramadan, les ceintures d'explosifs, [...]“ (das Gebet, den Ramadan, die Bombengürtel) in einer Assoziationskette direkt hintereinander stellt bzw. an das gleiche Verb „faire“ (machen) hängt (dies funktioniert in der deutschen Übersetzung leider nicht). Andererseits spiegelt sich darin Kamels Überzeugung wider, als,Araber' entweder zum Lachen zu bringen oder Angst einzuflößen und seinen vom Imam des Viertels unterstützten Entschluss, in seinen Shows mit den Ängsten des Publikums zu spielen und die wachsende Islamophobie als Kassenschlager zu missbrauchen. (Auch der Imam gibt Kamel ja folgenden Rat: „Les mécréants ils rient sur celui qu'ils ont peur, l'islam ils ont peur beaucoup, fais les peur contre l'islam ils vont très rire“ (Y. B. 2003, S. 17). ${ }^{18}$ ) Außerdem ist das Zitat ein gutes Beispiel für Benmilouds Strategie, Vorurteile und Missstände gleichzeitig anzusprechen und dadurch seine Gesellschaftskritik als zweischneidig zu präsentieren. Die im Zitat angesprochene Gewalt gegen bzw. Unterdrückung von Frauen in islamisch fundamentalistisch geprägten Gesellschaften ist ein Aspekt, der im Roman mehrmals kritisch thematisiert wird - allerdings auch, wie im vorangehenden Zitat, mit Blick darauf, dass sie im französischen Diskurs auch zur Unterstützung generell islamfeindlicher Diskurse angeführt wird. ${ }^{19}$ Mit Aussagen wie der folgenden unterstreicht Y. B. außerdem auf satirische Art und Weise, dass frauenfeindliche Diskurse nicht das Monopol islamisch geprägter Gesellschaften sind:

[...] c'est interdit de donner le permis de conduire aux femmes [en Arabie], et ça c'est un des avantages de la charia que même le Gaulois de base je pense

\footnotetext{
17 Eigene Übersetzung: Ich weiß ja, du fragst dich, ob ich [praktizierender] Moslem bin [fast so] wie mein Vater. Ob ich die Gebete und den Ramadan einhalte, Bombengürtel trage und - so wie alle illegal eingewanderten Imame - in Kellermoscheen beschnittene Minderjährige schände, sie mit ihrem Kopftuch ans Minarett fessle - und das alles trotz Sarkozy.

18 Sinngemäß: „Die Ungläubigen lachen über das, was sie ängstigt, vorm Islam haben sie viel Angst, mach ihnen Angst vorm Islam, sie werden sehr lachen“.

19 Ähnliche Mechanismen lägen laut Alec Hargreaves auch dem großen Erfolg mancher beurette-Romane bzw. Reportagen über Migrantinnen, die gegen gewaltsame Väter/Brüder ankämpfen, zugrunde. Ihre Zeugenberichte bildeten für eine westliche Leserschaft ,a veritable industry trading on hyped-up negative stereotypes of the Islamic world and its diaspora" (Hargreaves 2006, S. 48).
} 
qu'il est d'accord pour être contre les femmes au volant, non? (Y. B. 2003, S. 89). ${ }^{20}$

Die Ambivalenz der beiden Werke, die sich in diesen ersten Eindrücken deutlich zeigt und generell $\mathrm{zu}$ vielfältigen Interpretationen geführt hat, ${ }^{21}$ betrifft auch ihre Inszenierung von Männlichkeiten, der sich die folgende Analyse widmen soll. Wie schon beschrieben, sind die Protagonisten von La Haine und Allah Superstar junge Männer, die kaum Aussicht auf Bestätigung durch Arbeit, Verantwortung, eine eigene Wohnung oder eigene Familie haben. Dadurch nehmen sie keine aktive Rolle in der (französischen Mehrheits-Erwerbs-)Gesellschaft ein. In einer Gesellschaft aber, ,in der die individuelle Unabhängigkeit als Kardinaltugend gilt, bedeutet die Tatsache, ohne Zugang zur Arbeitswelt zu sein, ein äußerstes Maß an Fremdbestimmung“ (Khosrokhavar 2003, S. 94) und damit Entmännlichung (vgl. Loshitzky 2005, S. 145). Gleichzeitig, und gerade deshalb setzen die Protagonisten alles daran, ihren Status als gesellschaftliches,Nichts' (vgl. Y. B. 2003, S. 11) zu überwinden bzw. zu überspielen. Diese Bestrebung inszenieren beide Werke - auf unterschiedlichen Ebenen - mit Elementen der Parodie. Dies soll im Folgenden anhand jeweils eines aus einer Fülle an möglichen Beispielen skizziert werden.

Mangels anderer Betätigungsmöglichkeiten füllt in La Haine die Pflege der Ehre den Alltag der jungen Männer im banlieue. Der Film setzt dies durch die Haltung und Gestik der Protagonisten (Mackerposen, breitbeiniger Gang, Gerangel, Boxgesten in die Luft, körpersprachliche Provokationen in Richtung Polizei), ihren rauen Umgangston und regelmäßige Nahaufnahmen von Objekten wie Boxsäcken, einer Pistole und breiten Ringen filmisch in Szene. Die drei Freunde Vinz, Said und Hubert stehen dabei nicht auf gleicher Ebene, bzw. liegt ein besonderer Fokus auf der Beziehung zwischen Vinz (osteuropäisch-jüdischer Hintergrund) und $\mathrm{Hu}-$ bert (subsaharisch-christlicher Hintergrund). Ist Said (maghrebinisch-muslimischer Hintergrund) als kleingewachsener,Dampfplauderer' für Vinz weder Vorbild noch Konkurrent, so eifert er dem körperlich überlegenen, ruhigeren Hubert nach und schreibt ihm mitunter mehr,Street credits` zu, als dieser für sich verbuchen würde. So findet Hubert es beispielsweise ,,pas superglorieux“ (Kemmner 2001, S. 31), als Vinz sich mit einer ausstehenden Strafe für Taschendiebstahl vor ihm brüsten will, weil er annimmt, Hubert hätte als Schwarzer und gelegentlicher Drogendealer bereits Gefängniserfahrung. Huberts Körper, der durch Boxtraining gestählt und dem von Vinz und Said weitaus überlegen ist, fungiert im Film immer wieder als Männlichkeitssymbol per se. Zeigt die Kamera Vinz und Said hauptsächlich dabei, wie sie herumzappeln oder -sitzen, so wird Hubert etwa in einer Szene besonders lang (und in Slow-Motion) beim Boxen mit nacktem Oberkörper gezeigt (während Vinz beo-

\footnotetext{
20 Sinngemäß: In Saudi-Arabien ist es verboten, Frauen den Führerschein zu geben, und das ist einer der Vorteile der Scharia, ich denke, selbst der Standardgallier ist damit einverstanden, gegen Frauen am Steuer zu sein, nicht?

21 Die Stimmen reichen etwa bei La Haine von Jodie Fosters Interpretation als ,,a truly sincere and contemporary voice that [is] as explosive and strong as it [is] entertaining [and that] deals with violence in a way that's more mature than any film I've seen in the past ten years“ (Deussing 1996) zu „This film should be burnt" (Vincendeau 2005, S. 83), der Reaktion eines Jugendlichen im Zuge von Filmscreenings am Drehort in Chanteloup-les-Vignes (vgl. Tarr 2005, S. 64).
} 
bachtet, wie Huberts Muskeln spielen, er schwitzt, es in der Boxhalle dampft). Vinz versucht durch überzeichnete Posen seine Defizite gegenüber Hubert auszugleichen. Durch übertrieben breiten Gang, besonders rohes Vokabular, die wiederholte Geste der gezückten Pistole usw. performt er eine Art „Männlichkeit als Maskerade“ macht sich genau dadurch aber wiederholt lächerlich. ${ }^{22}$ Stellvertretend für eine Fülle möglicher Beispiele sei hier nur jene Sequenz erwähnt, die Vinz dabei zeigt, wie er - in Anlehnung an Travis Bickle (Robert de Niro) in Martin Scorseses Taxi Driver (1976) - vor dem Spiegel Gangsterposen übt und schließlich eine imaginäre Pistole zückt (Kemmer 2001, S. 17). Dies ist nur ein Beispiel für Kassovitz' komplexes Spiel mit intermedialen Verweisen und kollektiven Vorstellungen und eine komische und satirische Parodie zugleich: komisch, da Vinz seinen,Kraftakt ' durch das Kratzen und genaue Observieren seiner Nase unterbricht; satirisch, da er wenige Stunden später - im Gegensatz zu Travis Bickle in Taxi Driver - es nicht über sich bringt, mit einer zufällig gefundenen Polizeipistole den weißen Skinhead zu töten, der Said und Hubert angegriffen hat, sondern schließlich selbst von einem Polizisten erschossen wird. Dieses Ende ist bitter ironisch, da Vinz von Anfang an behauptet, aus Rache einen Polizisten zu erschießen, sollte Abdel seinen Verletzungen erliegen. Die Diskrepanz zwischen Posen und Taten ironisiert Vinz' Streben nach Härte und Männlichkeit und macht ihn zum Anti-Helden par excellence - er wird dadurch aber auch zu einem Sympathieträger.

In Allah Superstar manifestiert sich die Sehnsucht nach anerkannter Männlichkeit im Streben nach medialer Bekanntheit und damit Geld, Anerkennung und Erfolg bei den Frauen (besonders der angebeteten Nawal). Kamel will ,,au minimum star“ sein - aber ,pas pour la frime ou quoi, c'est pour la survie“ (Y. B. 2003, S. 11). ${ }^{23}$ Mehrmals betont Kamel, ihm sei Politik egal. Es gehe ihm nicht um kulturelle Wurzeln, sondern um die Äste, nicht um seine Herkunft, sondern um die Zukunft: „Ce qui m'intéresse c'est pas les racines c'est les branches [...] l'important c'est pas d'où tu viens mais où tu vas“ (Y. B. 2003, S. 48). Aus diesem Grund ist er auch taub für die Kritik seines Vorstadtfreunds Bala, der von Kamels Shows wenig begeistert ist und ihm vorwirft, er reproduziere damit koloniale Menschenzoos und spiele mit seinen Darstellungen den „weißen Medien“ die erwarteten Bilder aus dem banlieue in die Hände. ${ }^{24}$ Überlegungen dieser Art sind Kamel jedoch fremd, ihm geht es um Geld und Respekt (,Moi ce que je veux c’est le respect“( (Y. B. 2003, S. 31)), nicht um ideologische Beweggründe. Orientierungslos zwischen Kulturen und Ideo-

\footnotetext{
22 In der Rezeption des Films wird dieser Punkt übrigens häufig besonders kritisch aufgegriffen, da Kassovitz dadurch stereotype Bilder schwarzer Virilität und jüdischer,Feminisierung` wiederhole. Kritiker/ -innen sehen darin eine Reinszenierung der Klischees vom komplexbehafteten, feminisierten Juden und dem schwarzen, muskulösen Drogendealer und verweisen auf die Wiederholung rassistischer Klischees (so prüft Vinz im Spiegel immer wieder seine Nase, Hubert und Said sind beide Drogendealer). (Vgl. u. a. Moscowitz 2009, S. 14).

23 Meine Übersetzungen: Kamel will „,mindestens Star“ werden. „Nicht fürs Angeben und so, es geht ums Überleben".

24 Bala, Immigrant aus Mali, könnte man als Gegenkonstruktion zu Kamel sehen. Ist Kamel ideologisch und religiös quasi ein unbeschriebenes Blatt, ist Bala praktizierender Moslem. Während Kamel französischen Mediensternchen nacheifert, sind Balas Helden PoC-Widerstandskämpfer und -stars wie Frantz Fanon, Malcolm X, Martin Luther King, Bob Marley und Halle Berry.
} 
logien wird Kamel zum Spielball anderer: Nawal, sein französischer Produzent, die Medien, der Imam des Viertels, sie alle benutzen ihn für jeweils unterschiedliche finanzielle und strategische Interessen. Das Netz aus Satire, Parodie und Ironie ist im Roman besonders dicht und kann hier nicht im Detail herausgearbeitet werden. Ganz augenscheinlich basiert aber schon Kamels Kunstfigur, mit der er als Standup-Comedian zu zweifelhaftem Ruhm kommt, auf der Parodie von Djihadisten: Mit falschem Bart und falschen Bombengürteln ausgestattet spielt er Sketches, in denen er z. B. um Kulturförderung für Jeunesse et Djihad, ein djihadistisches Kinderlager, ansucht (Y. B. 2003, S. 41). ${ }^{25}$ Kamel wechselt ständig zwischen verschiedenen Rollen: Er erscheint als perspektivloser Fastfood-Verkäufer, unreflektierter Mitläufer im Umfeld seiner Freunde, als verblendeter Anbeter der vermeintlich keuschen Nawal (die ihm das Geld aus der Tasche zieht und gleichzeitig mit einem seiner Freunde schläft), als fanatischer Islamist in seiner Show und als provokanter Neuling in der französischen Comedy-Landschaft. Mit all diesen Rollen bedient er stereotype Vorstellungen von jungen Franko-Maghrebinern. Durch das ständige Wechseln zwischen diesen Rollen entblößt der Roman sie als soziale Konstrukte. Das SelbstmordAttentat am Ende gemahnt allerdings auch an die Gefahr solcher Zuschreibungen (vgl. Étienne Balibar zu Überdeterminierung und Frustpotential am Beginn dieses Artikels) und ironisiert gleichzeitig Kamels Streben nach einer Zukunft ohne lästige kulturelle Hintergründe auf bittere Art und Weise. ${ }^{26}$ Ähnlich wie La Haine endet auch Allah Superstar mit einem Knalleffekt, der die satirischen und slapstickartigen Aspekte der Werke in ein ernstes Licht rückt.

La Haine und Allah Superstar inszenieren ihre männlichen Protagonisten also als Marginalisierte, die nach Attributen,hegemonialer Männlichkeit‘ wie physischer Stärke oder finanziellem Erfolg streben, dabei aber scheitern bzw. sich permanent als Anti-Helden entblößen. Gleichzeitig zeigen die Werke aber auch, wie die Protagonisten von ihrem Umfeld in bestimmte Rollen gedrängt werden. Dadurch werden beide, das angestrebte Ideal der jungen Männer und das mediatisierte Bild der banlieusards, in Frage gestellt. Klischierte Männlichkeitsbilder werden so in ihrer Übertreibung hinterfragt - allerdings möglicherweise auch auf problematische Art und Weise zelebriert bzw. perpetuiert. Dies kann insofern bedenklich sein, als die Rezeptionsgeschichte beider Werke zeigt, wie sehr die Grenze zwischen Dokumentation und Fiktion bisweilen verschwimmen bzw. wie wenig das parodistische Element teilweise wahrgenommen wurde. So wurde zum Beispiel La Haine in seinem Erscheinungsjahr vom damaligen Premierminister Alain Juppé und seinem Team offiziell gescreent ${ }^{27}$ (vgl. Ruhe 2006, S. 108). Außerdem riefen öffentliche Filmscreenings in Vororten wie Chanteloup-les-Vignes, St. Denis oder Sarcelles heftige

\footnotetext{
25 Diese Art und Weise der Parodie erinnert an die Satire-Formate über die Kämpfer von Daesh/IS, die Ende 2014 im arabischen Fernsehen kursierten, z. B.: die libanesische Ktir Salbe Show (vgl. Verset 2014).

26 Mit Blick auf den Zulauf der Daesh/IS und seiner Verbrechen bzw. der Pariser Attentate im Jänner und November 2015 bekommt Y. B.s 2003 erschienene Satire diesbezüglich eine besonders alarmierende Dimension (vgl. Zerstörung von Weltkulturerbe im Irak).

27 Ironischerweise wurde so also ausgerechnet ein Film, dem von Kritiker/-innenseite mehrfach vorgeworfen wurde, soziale Probleme verflacht darzustellen und ästhetisch auszuschlachten (vgl. Moscowitz 2009, S. 14; Sharma 2000, S. 105), von politischer Seite als sozialhistorisches Dokument über die Ereignisse in den banlieues behandelt.
} 
Reaktionen unter dem (größtenteils jugendlichen) Publikum hervor: Mit lauten Zwischenrufen und demolierten Stühlen äußerten Zuseher/-innen ihren Unmut darüber, dass der Film sie falsch repräsentiere (Vincendeau 2005, S. 69, 82 f.). Auch auf Blogeinträgen zu Allah Superstar bezieht sich bisweilen die Kritik darauf, dass die (nie behauptete) Identität zwischen Autor und Protagonist des Romans bezweifelt wird (vgl. Kommentar BVIALLET auf babelio.com [2014]).

In beiden Werken bleibt auch dadurch letztendlich offen, wie man die Darstellung der Protagonisten deuten soll. Beide Werke stehen auf jeden Fall in starkem Kontrast zu Theorien harmonischer Multikulturalität, Third Space und Hybridität ${ }^{28}$, die sich überwiegend auf das Potential von Migrationsgesellschaften konzentrieren. Die französische Gesellschaft wird, ganz im Gegenteil, als sich im Krisenzustand befindlich präsentiert. Die Werke spiegeln ein soziales Klima in den französischen Großstadtvororten wider, das an einen ,durch den 11. September reaktivierten,clash of cultures“ à la Huntington“" (Porra 2009, S. 165) erinnert. Somit thematisieren sie (auch) ein politisches Problem: die Selbststilisierung Frankreichs als Republik der Menschenrechte und der Gleichheit, die jedoch verhältnismäßig blind ist für die Heterogenität der Nation und die Folgen ihrer kolonialen Vergangenheit bzw. mangelhafte Integrationspolitik.

Open access funding provided by University of Innsbruck.

Open Access Dieser Artikel wird unter der Creative Commons Namensnennung 4.0 International Lizenz (http://creativecommons.org/licenses/by/4.0/deed.de) veröffentlicht, welche die Nutzung, Vervielfältigung, Bearbeitung, Verbreitung und Wiedergabe in jeglichem Medium und Format erlaubt, sofern Sie den/die ursprünglichen Autor(en) und die Quelle ordnungsgemäß nennen, einen Link zur Creative Commons Lizenz beifügen und angeben, ob Änderungen vorgenommen wurden.

\section{Literatur}

Bachmann, Christian. 2002. Violences urbaines: Ascension et chute des classes moyennes à travers cinquante ans de politique de la ville. Paris: Hachette.

Balibar, Étienne. 2007. Uprisings in the Banlieue. Constellations 14(1):47-71. doi:10.1111/j.1467-8675. 2007.00422.x.

Beaud, Stéphane, und Michel Pialoux. 2003. Violences urbaines, violences sociale: genèse de nouvelles classes dangereuses. Paris: Hachette.

Bhaba, Homi. 1994. The Location of Culture. London: Routledge.

BVIALLET. 2014. Ce livre ne fait pas rire du tout. http://www.babelio.com/livres/Y-B-Allah-superstar/ 5486. Zugegriffen: 11.10.2014.

Connell, Raewyn. [1995] 2005. Masculinites, 2. Aufl., Cambridge: Polity Press.

Deussing, Ryan. 1996. Interview: Kassovitz, Foster. http://old.thing.net/ttreview/febrev.04.html (Erstellt: 2. 9. 1996). Zugegriffen: 1.11 .2014 .

Doering, Martin, und Dietmar Osthus. 2002. Black, Blanc, Beur: Metaphorische Identität, identische Metaphern? - Formen und Funktionen der Metaphorik in der französischen Tagespresse zum Mondial 1998. metaphorik.de 3:17-43.

Goudaillier, Jean-Pierre. 2001. Le dire des maux, les maux du dire. En guise d'introduction. In Comment tu tchatches!, Hrsg. Jean-Pierre Goudaillier, 8-43. Paris: Maisonneuve et Larose.

\footnotetext{
28 Hier sei nur am Rande auf Homi Bhabas v. a. in den Cultural Studies überaus erfolgreiche Theorien in Schriften wie The Location of Culture (1994) verwiesen.
} 
Hargreaves, Alec. 1999. No Escape? From 'cinéma beur' to the 'cinéma de la banlieue'. In Die Kinder der Immigration. Les enfants de l'immigration, Hrsg. Ernstpeter Ruhe, 115-128. Würzburg: Königshausen \& Neumann.

Hargreaves, Alec. 2006. Testimony, Co-Authorship, and Dispossession among Women of Maghrebi Origin in France. Research in African Literatures 37(1):42-54.

INSEE. Commune d'Évry. http://www.insee.fr/fr/themes/dossier_complet.asp?codgeo=COM-91228. Zugegriffen: 1.2.2016.

Kassovitz, Mathieu. 1995. La Haine. Aufzeichnung ARTE 29.10.2010 (OF).

Kemmer, Ernst (Hrsg.). 2001. La Haine. Scénario de Mathieu Kassovitz. Stuttgart: Reclam.

Khosrokhavar, Farhad. 2003. Die verschiedenen Formen muslimischer Religiosität in Frankreich. In Der Islam in Europa: Der Umgang mit dem Islam in Frankreich und Deutschland, Hrsg. Alexandre Escudier, 88-106. Göttingen: Wallenstein.

Kokoreff, Michel, Odile Steinauer, und Pierre Barron. 2007. Les émeutes urbaines à l'épreuve des situations locales SociologieS. http://sociologies.revues.org/254. Zugegriffen: 2.1.2016.

Loshitzky, Yosefa. 2005. The post-Holocaust Jew in the age of postcolonialism: La Haine revisited. Studies in French Cinema 5(2):137-147.

Moscowitz, David. 2009. ,You talkin’ to me?‘ Mediating Postmodern Blackface in La Haine. Critical Studies in media Communication 26(1):1-18.

Ottersbach, Markus. 2004. Jugendliche in marginalisierten Quartieren. Ein deutsch-französischer Vergleich. Wiesbaden: Verlag für Sozialwissenschaften.

O'Dowd-Smyth, Christine. 2004. Development in Diasporic North African Literature of French Expression: The ,Indeterminacy of Diasporic Identity ' and the Second generation of Immigration. In Contemporary French Cultures and Societies, Hrsg. Frédéric Royall, 315-336. Bern: Peter Lang.

Porra, Véronique. 2009. Risse in der Mimesis - Bemerkungen zur romanesken Darstellung des 11. September 2001 in der französischen Literatur. In 9/11 als kulturelle Zäsur. Repräsentationen des 11. September 2001 in kulturellen Diskursen, Literatur und visuellen Medien, Hrsg. Sandra Poppe, Thorsten Schüler, und Sascha Seiler, 163-182. Bielefeld: transcript.

Ruhe, Cornelia. 2006. Cinéma beur. Analysen zu einem neuen Genre des französischen Films. Konstanz: UVK.

Sangsue, Daniel. 1994. La Parodie. Paris: Hachette.

Sharma, Sanjay, und Ashwani Sharma. 2000. ,So far so good...' La Haine and the poetics of the Everyday. Theory Culture Society 17(103):103-116.

Spindler, Susanne. 2009. Im Netz hegemonialer Männlichkeit. Männlichkeitskonstruktionen junger Migranten. In Dimensionen der Kategorie Geschlecht: Der Fall Männlichkeit, 2. Aufl., Hrsg. Mechthild Bereswill, Michael Meuser, und Sylka Scholz, 119-135. Münster: Westfälisches Dampfboot.

Tarr, Carrie. 2005. Reframing difference: Beur and banlieue filmmaking in France. Manchester: MUP.

Verset, Jean-Claude. 2014. L'Etat Islamique ridiculisé par des vidéos satiriques arabes. http://tinyurl.com/ 19lor6u (Erstellt: 3. 9. 2014). Zugegriffen: 5.12.2014.

Vincendeau, Ginette. 2005. La Haine (Mathieu Kassovitz, 1995). London: Tauris.

Vitali, Ilaria. 2011. Intrangers II: Littérature beur, de l'écriture à la traduction. Paris: L'Harmattan.

Weil, Patrick. 1995. La France et ses étrangers: L'aventure d'une politique de l'immigration de 1938 à nos jours. Paris: Gallimard.

Y. B. 2003. Allah superstar. Paris: Grasset.

Laura Fuchs-Eisner MMag.a, ist DOC-Stipendiatin der Österreichischen Akademie der Wissenschaften mit einem Dissertationsprojekt zu Satire und Terrorismus in französischsprachigen Romanen, Filmen und Graphic Novels nach 9/11 (betreut an der Romanistik Innsbruck und Wien). Nach dem Studium der Vergleichenden Literaturwissenschaft und des Lehramts für Englisch und Französisch an der Universität Wien war sie von 2014 bis 2016 wissenschaftliche Mitarbeiterin an der Romanistik Innsbruck. 